\title{
Design, Synthesis, and Evaluation of Potential Carbamate Prodrugs of 5'-methylthioadenosine (MTA)*
}

\section{Ashwini Sadanand Ranade}

Rutgers University Ernest Mario School of Pharmacy

Joseph R Bertino

Rutgers Cancer Institute of New Jersey

Longqin Hu ( $\sim$ longhu@pharmacy.rutgers.edu )

Rutgers University Ernest Mario School of Pharmacy https://orcid.org/0000-0002-1799-5652

\section{Research Article}

Keywords: 5'-Methylthioadenosine (MTA), carbamate prodrugs, N-(alkyloxy)carbonyl-MTA, carboxyesterase, antimetabolite

Posted Date: March 8th, 2021

DOl: https://doi.org/10.21203/rs.3.rs-272240/v1

License: (a) (1) This work is licensed under a Creative Commons Attribution 4.0 International License. Read Full License 


\section{Abstract}

5'-Methylthioadenosine (MTA) is a natural substrate of MTA phosphorylase (MTAP) and is converted to adenine via a salvage pathway for AMP production in normal healthy cells. The lack of MTAP expression in many solid tumors and hematologic malignancies compared to normal healthy cells has been explored in a potential therapeutic strategy to selectively target tumor cells using antimetabolites such as 5fluorouracil (5-FU) and 6-thioguanine (6-TG) while protecting normal healthy cells with MTA. Herein, a series of carbamate prodrugs, namely the $\mathrm{N}$-(alkyloxy)carbonyl-MTA derivatives $\mathbf{2 a - f}$, was designed, synthesized, and evaluated as potential prodrugs of MTA. All carbamate prodrugs were stable in phosphate buffer, $\mathrm{pH} 7.4$ at $37^{\circ} \mathrm{C}$. In the presence of mouse liver microsomes, the prodrugs were converted to MTA at varying rates with the hexyl and butyl carbamates $\mathbf{2 a}$ and $\mathbf{2 b}$ most readily activated $\left(t_{1 / 2}\right.$ of 1.2 and $9.4 \mathrm{~h}$, respectively). The activation was shown to be mediated by carboxyesterases present in mouse liver microsomes.

\section{Introduction}

5'-Methylthioadenosine (MTA) is a byproduct formed during polyamine biosynthesis [1] and is a substrate of methylthioadenosine phosphorylase (MTAP) in a process that generates adenine and 5methylthioribose-1-phosphate [2,3]. The latter is then converted in several steps to methionine in the methionine salvage pathway. Adenine is converted to AMP by the ubiquitous enzyme adenine phosphoribosyltransferase (APRT) with phosphoribosyl-1-pyrophosphate (PRPP) serving as the donor of the phosphoribosyl group. Although AMP may also be produced in cells by de novo purine biosynthesis, MTAP is responsible for the generation of essentially all free adenine in normal healthy cells [4].

Many solid tumors and hematologic malignancies are characterized by a deficiency of MTAP and are unable to salvage adenine from MTA. The difference in the lack of expression of MTAP in tumor cells compared to normal healthy cells has been explored in a therapeutic strategy for selectively killing tumor cells $[5,4]$. Antimetabolites such as 5-fluorouracil (5-FU), 6-thioguanine (6-TG) or antifolates disrupt DNA replication and inhibit de novo purine synthesis through the release of cytotoxic nucleotides generated via phosphorylation using phosphoribosyl-1-pyrophosphate (PRPP). One major drawback of these cytotoxic nucleotides is that they produce harmful side effects on the growth and proliferation of replicating normal healthy cells. To reduce the cytotoxicity of these chemotherapeutic drugs and thus increase their therapeutic index, administration of MTA or MTA analogs in conjugation with these antimetabolites could prevent damage to healthy MTAP-competent cells [6, 7]. This is because adenine generated in the above adenine salvage pathway by MTAP in healthy cells will block the conversion of 5-FU/6-TG to their cytotoxic nucleotides by competing for the rate-limiting pools of PRPP. Since no adenine is produced in tumor cells due to lack of MTAP, PRPP is present in sufficient levels and the co-administered drug can be readily converted to its toxic metabolite. Thus, a high degree of selectivity can be achieved. Indeed, MTA protection was shown to be quite remarkable in vitro with at least 10 - to 100 - fold changes in $I C_{50}$ values when co-administered with 6-TG, suggesting that higher doses of 6-TG than currently administered could be given before any side effects are seen $[3-5,8,9]$. MTA in high doses has been shown to prevent liver 
damage in experimental models $[10,11]$. Bertino and coworkers also reported that co-administration of $100 \mathrm{mg} / \mathrm{kg}$ MTA protected nude mice from a lethal dose of 5-FU, raising its maximum tolerated dose from 75 to $200 \mathrm{mg} / \mathrm{kg}$ [5]. In a clinical study, MTA was dosed daily at $600 \mathrm{mg}$ for one month to 50 volunteers and at $1600 \mathrm{mg}$ daily for one month to another 10 volunteers without reports of any toxicity in either group $[12,13]$. These proof of concept preclinical and clinical studies suggest that MTA can produce a beneficial protective effect to healthy cells and thus reduce systemic side effects during cancer chemotherapy using these antimetabolites.

There are several MTA prodrugs disclosed in a US patent by modifying the ribosyl hydroxyl groups in the form of carbamates, phosphates and esters to deliver MTA to protect MTAP-competent cells [14]. Herein, we report the design of new prodrugs of MTA by attaching promoieties to the 6-amino group of adenine for activation by carboxylesterases to release MTA in an effort to overcome the potential stability and bioavailability issues of MTA.

\section{Results And Discussion}

\section{Prodrug Design}

We focused our design of prodrugs on the 6- $\mathrm{NH}_{2}$ of MTA that could potentially be activated by a carboxylesterase enzyme $[15,16]$. Carboxyesterases are ubiquitously present in most animals including humans, and several types are present in blood, liver and other tissues. The initial design of our MTA prodrugs was based on the activation of the marketed prodrug of 5-FU known as capecitabine. Clinically, capecitabine passes intact through the intestinal mucosa and is converted to its active form, 5-FU, by a 3step enzymatic activation in liver and tumor tissues. Its activation is initiated by carboxylesterasemediated hydrolysis of the carbamate moiety to 5'-deoxy-5-fluorocytidine primarily in the liver with a reported half-life of 0.55 to 0.87 hours $[17,18]$.

We used a similar strategy to design the carbamate or $\mathrm{N}$-(alkyloxy)carbonyl-MTA prodrugs 2a-f as depicted in Figure 1 that can potentially be activated by the liver carboxylesterases for systemic release of MTA. In the process of designing MTA carbamate prodrugs $2 \mathrm{a}-\mathrm{f}$, we varied the alkyl groups of the promoiety from 2 to 6 carbons to discern what effect the alkyl group would have on the rate of cleavage of the carbamate moiety by the liver carboxylesterase. The carbamate functional group can impart chemical stability and since carbamates do not ionize, they can also provide better oral bioavailability for the prodrugs. Upon hydrolysis, the prodrugs would release alcohol and carbamic acid that would be unstable under physiological $\mathrm{pH}$ and decompose spontaneously to the parent amine drug (in our case, MTA) and $\mathrm{CO}_{2}$.

\section{Chemical Synthesis}

The syntheses of $N$-(alkyloxy)carbonyl-MTA prodrugs $\mathbf{2 a - f}$ are straightforward and were accomplished in two steps as shown in Scheme 1. In step 1, treatment of commercially available MTA with an excess amount (6 eq.) of commercially available alkyl chloroformates in the presence of a base in anhydrous 
dichloromethane gave the intermediates 3a-f with a carbamate on 6- $\mathrm{NH}_{2}$ of adenine and two carbonates on the two hydroxyl groups of ribose. The carbonates were more easily and selectively hydrolyzed using $0.1 \mathrm{~N} \mathrm{NaOH}$ to afford the desired prodrugs $2 \mathrm{a}-\mathrm{f}$.

\section{Evaluation of $\mathrm{N}$-(alkyloxy)carbonyl-MTA Prodrugs 2a-f}

We first evaluated the stability of the carbamate or $N$-(alkyloxy)carbonyl-MTA prodrugs $2 \mathrm{a}-\mathrm{f}$ in $50 \mathrm{mM}$ phosphate buffer, $\mathrm{pH} 7.4$ at $37^{\circ} \mathrm{C}$ using an HPLC assay that is capable of separating the prodrugs and the parent molecule MTA on a reverse phase $\mathrm{C}_{18}$ column. As shown in Table 2 , all the $\mathrm{N}$ (alkyloxy)carbonyl-MTA prodrugs 2a-f of MTA demonstrated great stability under these conditions with less than $10 \%$ change in measured peak area over 72 hours. Then we determined their activation in the presence of mouse or human liver microsomes. In the presence of mouse liver microsomes, the prodrugs were activated at varying rates in the order of hexyl $(\mathbf{2} \mathbf{a})>$ butyl $(\mathbf{2} \mathbf{b})>\operatorname{propyl}(\mathbf{2} \mathbf{e})>2$-methoxyethyl $(\mathbf{2} \mathbf{f})>$ isopropyl $(\mathbf{2 c}) \approx$ ethyl $(\mathbf{2 d})$ with the hexyl and butyl carbamate prodrugs $\mathbf{2} \mathbf{a}$ and $\mathbf{2} \mathbf{b}$ exhibiting a half-life of 1.2 and $9.4 \mathrm{~h}$, respectively. As shown in Figure $2 \mathrm{~A}$ and $2 \mathrm{~B}$, the activation of prodrugs $\mathbf{2 a}$ and $\mathbf{2 b}$ in the presence of MLM was accompanied with the formation of MTA, parent drug. It is also clear from the MTA decomposition phase shown

in Figure $2 \mathrm{~B}$ vs $2 \mathrm{~A}$ that overall degradation of MTA was slower when the prodrug activation was slower in the presence of MLM, suggesting that prodrug $\mathbf{2} b$ significantly increased the stability of MTA which validates our design principle. However, as shown in Table 2, the activation of the $N$-(alkyloxy)carbonylMTA prodrugs in the presence of human liver microsomes (HLM) was much slower with $t_{1 / 2}$ all greater than $48 \mathrm{~h}$; prodrugs $\mathbf{2 a}$ and $\mathbf{2 b}$ showed about $44 \%$ and $19 \%$ change, respectively, in the peak area of the prodrugs measured over the 48-h incubation with HLM.

To confirm the type of enzyme involved in the metabolic activation of $N$-(alkyloxy)carbonyl-MTA prodrugs, we performed the activation of $2 \mathrm{~b}$ using mouse or human liver microsomes in the presence of 1 $\mathrm{mM}$ bis(4-nitrophenyl)-phosphate (BNPP), an inhibitor of carboxyesterases, or $1 \mathrm{mM}$ of nicotinamide adenine dinucleotide phosphate (NADPH), a cofactor needed for oxidative metabolism. As shown in Figure 3 , the BNPP completely prevented the activation of $\mathbf{2} \mathbf{b}$ by mouse liver microsomes while NADPH had no effect on the activation of $\mathbf{2 b}$, confirming that the activation of $N$-(alkyloxy)carbonyl-MTA prodrug $\mathbf{2} \mathbf{b}$ is mediated by a carboxypeptidase(s), not by oxidoreductases, in mouse liver microsomes. Also consistent with the earlier results, the slow activation of $\mathbf{2} \mathbf{b}$ observed in the presence of human liver microsomes is only marginally affected by the addition of BNPP or NADPH.

In summary, we designed, synthesized, and conducted activation studies of carbamate prodrugs of 5'methylthioadeosine (MTA), in the form of $N$-(alkyloxy)carbonyl-MTA. All of the carbamate prodrugs obtained were stable in phosphate buffer, $\mathrm{pH} 7.4$ at $37^{\circ} \mathrm{C}$. In the presence of mouse liver microsomes, the prodrugs were converted to MTA at varying rates with the hexyl and butyl carbamates $\mathbf{2 a}$ and $\mathbf{2} \mathbf{b}$ most readily activated exhibiting $t_{1 / 2}$ of 1.2 and $9.4 \mathrm{~h}$, respectively. The activation was shown to be mediated by carboxyesterases present in mouse liver microsomes. The activation of these prodrugs in the presence 
of human liver microsomes was much slower with $\mathrm{t}_{1 / 2}$ all greater than $48 \mathrm{~h}$. Consistent with the activation studies in the presence of mouse liver microsomes, the hexyl and butyl carbamates $\mathbf{2} \mathbf{a}$ and $\mathbf{2} \mathbf{b}$ were shown to have the highest level of conversion at $44 \%$ and $19 \%$, respectively. Further studies will be needed to fully explore the potential of these carbamate prodrugs.

\section{Experimental Section}

All reactions were performed in oven-dried glassware. All solvents used were either ACS reagent grade or HPLC grade. All reactions were performed using anhydrous solvents. Moisture sensitive reactions were performed under $\mathrm{N}_{2}$ atmosphere. Air sensitive reagents were transferred to a reaction flask using a cannula under $\mathrm{N}_{2}$ atmosphere. All reactions were monitored by Thin Layer Chromatography (TLC) using $0.25 \mathrm{~mm}$ Whatmann precoated silica gel plates. TLC plates were visualized by either UV absorbance or potassium permanganate solution. Purification was done by using automated flash column chromatography using Teledyne ISCO CombiFlash Companion System.

Analytical LC-MS was obtained using Agilent 1200 Series LC system equipped with G6140A Quadruple MS on a $\mathrm{C}_{18}$ column. Solvent A was $0.1 \%$ formic acid $/ \mathrm{H}_{2} \mathrm{O}$ and solvent $\mathrm{B}$ was $0.1 \%$ formic acid in methanol and the gradient used was $10-90 \%$ in 5 mins at a flow rate of $0.8 \mathrm{ml} / \mathrm{min}$. NMR spectra were recorded on a Bruker Ultrashield $400 \mathrm{MHz}$ at ambient temperature. High Resolution Mass Spectrometry data was obtained at the Center for Integrative Proteomics Research (CIPR), Rutgers University.

\section{General procedure for the synthesis of N-(alkyloxy)carbonyl-MTA prodrugs (2a-f)}

5'-Methylthioadenosine (MTA) was suspended in 10-20 ml anhydrous dichloromethane. 1Methylimidazole (8 eq.) was added and the suspension was stirred at $0{ }^{\circ} \mathrm{C}$ for 5 mins. An alkyl chloroformate ( 6 eq.) was added dropwise to the cold suspension over 5 mins while maintaining the temperature at $0{ }^{\circ} \mathrm{C}$. The reaction mixture was allowed to warm to room temperature and was stirred for an additional 3 days. The reaction progress was monitored using TLC and LCMS. The reaction mixture was diluted with dichloromethane $(20 \mathrm{~mL})$ and washed successively twice each with saturated $\mathrm{NaHCO}_{3}$ $(10 \mathrm{ml})$ and brine $(10 \mathrm{~mL})$. The organic phase was separated and dried over $\mathrm{Na}_{2} \mathrm{SO}_{4}$ and filtered. The solvent was evaporated, and the crude product was purified by column chromatography using methanol and dichloromethane as an eluent to give the intermediate $3 a-f$ as an oil.

To a solution of intermediate $3 \mathrm{a}-\mathrm{f}$ in $5 \mathrm{~mL}$ ethanol was added $0.1 \mathrm{~N} \mathrm{NaOH}$ (1 eq.) and the resulting reaction mixture was stirred at room temperature for 12 hours. On completion of the reaction, $1 \mathrm{~N} \mathrm{HCl}$ was added to the reaction mixture until the $\mathrm{pH}$ was neutral. Dichloromethane $(10 \mathrm{~mL})$ was then added to the reaction mixture followed by washing with brine $(5 \mathrm{~mL})$. The organic layer was dried over $\mathrm{Na}_{2} \mathrm{SO}_{4}$, filtered and the solvent was evaporated. The crude product was purified using column chromatography in methanol in dichloromethane to give final target compounds $2 \mathrm{a}-\mathrm{f}$ as white solids. 
$N$-(hexoxycarbonyl)-2',3'-bis(hexylcarbonate)-MTA (3a): Yield, $140 \mathrm{mg}$ (61\%) obtained from $100 \mathrm{mg}$ (0.336 mmol) of MTA. ${ }^{1} \mathrm{H}$ NMR (400 MHz, Methanol- $\left.d_{4}\right) \delta(\mathrm{ppm}): 8.64(\mathrm{~s}, 1 \mathrm{H}), 8.50(\mathrm{~s}, 1 \mathrm{H}), 6.34(\mathrm{~d}, J=5.3 \mathrm{~Hz}$, $1 \mathrm{H}), 6.09(\mathrm{t}, J=5.5 \mathrm{~Hz}, 1 \mathrm{H}), 5.64(\mathrm{dd}, J=5.7,4.7 \mathrm{~Hz}, 1 \mathrm{H}), 4.46(\mathrm{td}, J=5.9,4.6 \mathrm{~Hz}, 1 \mathrm{H}), 4.26(\mathrm{t}, J=6.7 \mathrm{~Hz}$, 2H), $4.23-4.01(\mathrm{~m}, 4 \mathrm{H}), 3.11-2.90(\mathrm{~m}, 2 \mathrm{H}), 2.11(\mathrm{~s}, 3 \mathrm{H}), 1.81-1.65(\mathrm{~m}, 4 \mathrm{H}), 1.60(\mathrm{dq}, J=8.0,6.5 \mathrm{~Hz}$, $2 \mathrm{H}), 1.51-1.23(\mathrm{~m}, 18 \mathrm{H}), 0.98-0.87(\mathrm{~m}, 9 \mathrm{H}) ;{ }^{13} \mathrm{C}$ NMR $\left(100 \mathrm{MHz}\right.$, Methanol- $\left.d_{4}\right) \delta(\mathrm{ppm}): 154.2,153.9$, $152.2,152.1,151.1,150.0,142.9,122.4,86.3,81.8,75.5,75.4,68.8,68.6,65.7,35.5,33.4,31.3,31.2,31.2$, $30.9,28.5,28.3,28.2,27.9,25.4,25.3,25.1,25.0,24.7,22.3,22.3,22.2,22.1,15.2,13.1,13.1,13.0$; LCMS $\left(\mathrm{ESI}^{+}\right): 682.3[\mathrm{M}+\mathrm{H}]^{+}$.

$N$-(hexoxycarbonyl)-MTA (2a, LH1201): Yield, $67 \mathrm{mg}$ (77\%) obtained from $140 \mathrm{mg}(0.205 \mathrm{mmol})$ of 3a. ${ }^{1} \mathrm{H}$ NMR (400 MHz, Methanol- $\left.d_{4}\right) \delta(p p m): 8.63(\mathrm{~s}, 1 \mathrm{H}), 8.55(\mathrm{~s}, 1 \mathrm{H}), 6.11(\mathrm{~d}, \mathrm{~J}=5.0 \mathrm{~Hz}, 1 \mathrm{H}), 4.38(\mathrm{t}, \mathrm{J}=4.9$ $\mathrm{Hz}, 1 \mathrm{H}), 4.29,(\mathrm{t}, \mathrm{J}=6.2 \mathrm{~Hz}, 2 \mathrm{H}), 4.27(\mathrm{~m}, 1 \mathrm{H}), 2.98(\mathrm{dd}, \mathrm{J}=14.1,5.6 \mathrm{~Hz}, 1 \mathrm{H}), 2.90(\mathrm{dd}, \mathrm{J}=14.1,6.1 \mathrm{~Hz}$, 1H), $2.14(\mathrm{~s}, 3 \mathrm{H}), 1.85-1.69(\mathrm{~m}, 2 \mathrm{H}), 1.48(\mathrm{~s}, 2 \mathrm{H}), 1.42-1.31(\mathrm{~m}, 4 \mathrm{H}), 0.95(\mathrm{t}, \mathrm{J}=7.2 \mathrm{~Hz}, 3 \mathrm{H}) ;{ }^{13} \mathrm{C} \mathrm{NMR}$ $\left(100 \mathrm{MHz}\right.$, Methanol- $\left.d_{4}\right) \delta(\mathrm{ppm}): 152.7,151.3,150.6,149.0,143.1,121.7,89.1,84.3,73.6,72.6,66.1$, 36.1, 31.2, 28.5, 25.2, 22.2, 15.2, 13.0; HRMS (ESI+) m/z calcd for $\mathrm{C}_{18} \mathrm{H}_{28} \mathrm{~N}_{5} \mathrm{O}_{5} \mathrm{~S}[\mathrm{M}+\mathrm{H}]^{+}:$: 426.1806, found: 426.1816 .

$N$-(butoxycarbonyl)-2',3'-bis(butylcarbonate)-MTA (3b): Yield, $130 \mathrm{mg}$ (65\%) obtained from $100 \mathrm{mg}$ (0.336 mmol) of MTA. ${ }^{1} \mathrm{H}$ NMR $\left(400 \mathrm{MHz}, \mathrm{CDCl}_{3}\right) \delta(\mathrm{ppm}): 9.18(\mathrm{~s}, 1 \mathrm{H}), 8.75(\mathrm{~s}, 1 \mathrm{H}), 8.20(\mathrm{~s}, 1 \mathrm{H}), 6.20(\mathrm{~d}, J=5.4$ $\mathrm{Hz}, 1 \mathrm{H}), 6.04(\mathrm{t}, J=5.4 \mathrm{~Hz}, 1 \mathrm{H}), 5.60-5.55(\mathrm{~m}, 1 \mathrm{H}), 5.27(\mathrm{~s}, 1 \mathrm{H}), 4.47(\mathrm{q}, J=5.5 \mathrm{~Hz}, 1 \mathrm{H}), 4.26(\mathrm{t}, J=6.7$ $\mathrm{Hz}, 2 \mathrm{H}), 4.22-4.01(\mathrm{~m}, 4 \mathrm{H}), 3.05-2.85(\mathrm{~m}, 2 \mathrm{H}), 2.10(\mathrm{~s}, 3 \mathrm{H}), 1.73-1.52(\mathrm{~m}, 6 \mathrm{H}), 1.47-1.26(\mathrm{~m}, 6 \mathrm{H})$, $0.97-0.83(\mathrm{~m}, 9 \mathrm{H})$; LCMS $\left(E S I^{+}\right): 598.2[\mathrm{M}+\mathrm{H}]^{+}$.

$N$-(butoxycarbonyl)-MTA (2b, LH1205): Yield, $60 \mathrm{mg}(69 \%)$ obtained from $130 \mathrm{mg}(0.218 \mathrm{mmol})$ of $3 \mathbf{3 b} .{ }^{1} \mathrm{H}$ NMR (400 MHz, Methanol- $\left.d_{4}\right) \delta(\mathrm{ppm}):{ }^{1} \mathrm{H}$ NMR $\left(400 \mathrm{MHz}\right.$, Methanol- $\left.d_{4}\right) \delta(\mathrm{ppm}): 8.63(\mathrm{~s}, 1 \mathrm{H}), 8.55(\mathrm{~s}$, $1 \mathrm{H}), 6.11(\mathrm{~d}, J=5.0 \mathrm{~Hz}, 1 \mathrm{H}), 4.38(\mathrm{~s}, 1 \mathrm{H}), 4.30(\mathrm{t}, J=6.6 \mathrm{~Hz}, 2 \mathrm{H}), 4.27-4.23(\mathrm{~m}, 1 \mathrm{H}), 2.97(\mathrm{~d}, J=5.6 \mathrm{~Hz}$, $1 \mathrm{H}), 2.92(\mathrm{~d}, J=6.1 \mathrm{~Hz}, 1 \mathrm{H}), 2.14(\mathrm{~s}, 3 \mathrm{H}), 1.76(\mathrm{~s}, 2 \mathrm{H}), 1.51(\mathrm{~d}, J=7.6 \mathrm{~Hz}, 2 \mathrm{H}), 1.01(\mathrm{t}, J=7.4 \mathrm{~Hz}, 3 \mathrm{H}) ;{ }^{13} \mathrm{C}$ $\operatorname{NMR}\left(125 \mathrm{MHz}\right.$, Methanol- $\left.d_{4}\right) \delta(\mathrm{ppm}): 152.5,151.5,151.3,149.6,142.8,122.2,89.1,84.3,73.5,72.7$, $65.5,36.1,30.6,18.7,15.1,12.6$; HRMS (ESI+) $\mathrm{m} / \mathrm{z}$ calcd for $\mathrm{C}_{16} \mathrm{H}_{24} \mathrm{~N}_{5} \mathrm{O}_{5} \mathrm{~S}[\mathrm{M}+\mathrm{H}]^{+}: 398.1493$, found: 398.1479 .

N-(Isopropoxycarbonyl)-2',3'-bis(isopropylcarbonate)-MTA (3c): Yield, $190 \mathrm{mg}$ (52\%) obtained from 200 mg $(0.67 \mathrm{mmol})$ of MTA. ${ }^{1} \mathrm{H}$ NMR $\left(400 \mathrm{MHz}\right.$, Methanol- $\left.d_{4}\right) \delta(\mathrm{ppm}): 8.52(\mathrm{~s}, 1 \mathrm{H}), 8.38(\mathrm{~s}, 1 \mathrm{H}), 6.19(\mathrm{~d}, J=$ $5.3 \mathrm{~Hz}, 1 \mathrm{H}), 5.96(\mathrm{t}, J=5.5 \mathrm{~Hz}, 1 \mathrm{H}), 5.54-5.49(\mathrm{~m}, 1 \mathrm{H}), 4.98(\mathrm{dt}, J=12.5,6.2 \mathrm{~Hz}, 1 \mathrm{H}), 4.81(\mathrm{dd}, J=12.5$, $6.2 \mathrm{~Hz}, 1 \mathrm{H}), 4.67(\mathrm{dt}, J=12.5,6.2 \mathrm{~Hz}, 1 \mathrm{H}), 4.33(\mathrm{q}, J=5.9 \mathrm{~Hz}, 1 \mathrm{H}), 2.92(\mathrm{dd}, J=14.3,5.8 \mathrm{~Hz}, 1 \mathrm{H}), 2.86(\mathrm{dd}$, $J=14.3,6.1 \mathrm{~Hz}, 1 \mathrm{H}), 2.00(\mathrm{~s}, 3 \mathrm{H}), 1.17$ (ddd, $J=46.6,20.1,6.3 \mathrm{~Hz}, 18 \mathrm{H}) ;{ }^{13} \mathrm{C}$ NMR $(100 \mathrm{MHz}$, Methanol$\left.d_{4}\right) \delta(\mathrm{ppm}): 155.0,154.7,153.6,153.1,152.5,151.4,144.3,123.8,87.7,83.2,76.8,76.6,74.5,74.2,71.0$, 37.0, 22.4, 16.7; LCMS $\left(E S I^{+}\right): 556.3[\mathrm{M}+\mathrm{H}]^{+}$. 
$N$-(Isopropoxycarbonyl)-MTA (2c, LH1206): Yield, $72 \mathrm{mg}$ (62\%) obtained from $170 \mathrm{mg}(0.3 \mathrm{mmol})$ of 3c. ${ }^{1} \mathrm{H}$ NMR $\left(400 \mathrm{MHz}\right.$, Methanol- $\left.d_{4}\right) \delta(\mathrm{ppm}): 10.38$ (bs, 1H), $8.67(\mathrm{~s}, 1 \mathrm{H}), 8.64(\mathrm{~s}, 1 \mathrm{H}), 6.01$ (d, J = $5.8 \mathrm{~Hz}$, 1H), $5.59(\mathrm{~s}, 1 \mathrm{H}), 5.39(\mathrm{~s}, 1 \mathrm{H}), 4.93$ (hept, $J=6.2 \mathrm{~Hz}, 1 \mathrm{H}), 4.83-4.77(\mathrm{~m}, 1 \mathrm{H}), 4.19(\mathrm{~s}, 1 \mathrm{H}), 4.11-4.05(\mathrm{~m}$, $1 \mathrm{H}), 2.91(\mathrm{dd}, J=13.9,5.9 \mathrm{~Hz}, 1 \mathrm{H}), 2.81(\mathrm{dd}, J=13.9,6.9 \mathrm{~Hz}, 1 \mathrm{H}), 2.06(\mathrm{~s}, 3 \mathrm{H}), 1.28(\mathrm{~d}, J=6.2 \mathrm{~Hz}, 6 \mathrm{H}) ;{ }^{13} \mathrm{C}$ NMR $\left(100 \mathrm{MHz}\right.$, Methanol- $\left.d_{4}\right) \delta$ (ppm): 152.5, 150.7, 150.6, 149.6, 141.5, 122.1, 89.7, 84.3, 74.6, 73.1, 70.3, 36.8, 21.9, 21.8, 16.7; HRMS (ESI+) $\mathrm{m} / \mathrm{z}$ calcd for $\mathrm{C}_{15} \mathrm{H}_{22} \mathrm{~N}_{5} \mathrm{O}_{5} \mathrm{~S}[\mathrm{M}+\mathrm{H}]^{+}: 384.1336$, found: 384.1350 .

N-(Ethoxycarbonyl)-2',3'-bis(ethylcarbonate)-MTA (3d): Yield, $197 \mathrm{mg}$ (57\%) obtained from $200 \mathrm{mg}$ (0.67 mmol) of MTA. ${ }^{1} \mathrm{H}$ NMR (400 MHz, Methanol- $\left.d_{4}\right) \delta(\mathrm{ppm}): 8.60(\mathrm{~s}, 1 \mathrm{H}), 8.47(\mathrm{~s}, 1 \mathrm{H}), 6.29(\mathrm{~d}, J=5.3 \mathrm{~Hz}$, $1 \mathrm{H}), 6.04(\mathrm{t}, J=5.4 \mathrm{~Hz}, 1 \mathrm{H}), 5.60(\mathrm{t}, J=5.1 \mathrm{~Hz}, 1 \mathrm{H}), 4.41(\mathrm{q}, J=5.7 \mathrm{~Hz}, 1 \mathrm{H}), 4.28(\mathrm{q}, J=7.1 \mathrm{~Hz}, 2 \mathrm{H}), 4.23-$ $4.17(\mathrm{~m}, 2 \mathrm{H}), 4.10(\mathrm{dd}, J=14.7,7.2 \mathrm{~Hz}, 2 \mathrm{H}), 2.99(\mathrm{dd}, J=14.4,5.8 \mathrm{~Hz}, 1 \mathrm{H}), 2.93(\mathrm{dd}, J=14.3,6.2 \mathrm{~Hz}, 1 \mathrm{H})$, $2.06(\mathrm{~s}, 3 \mathrm{H}), 1.32(\mathrm{t}, J=7.1 \mathrm{~Hz}, 3 \mathrm{H}), 1.28(\mathrm{t}, J=7.1 \mathrm{~Hz}, 3 \mathrm{H}), 1.19(\mathrm{t}, J=7.1 \mathrm{~Hz}, 3 \mathrm{H}) ;{ }^{13} \mathrm{C} \mathrm{NMR}(100 \mathrm{MHz}$, Methanol- $\left.d_{4}\right) \delta(\mathrm{ppm}): 153.4,153.1,151.5,151.4,150.4,149.3,142.2,121.8,85.7,81.1,74.7,74.7,64.0$, $63.8,60.9,34.8,14.4,12.7,12.5,12.3$; LCMS $\left(E S I^{+}\right): 514.5\left[\mathrm{M}+\mathrm{H}^{+}\right.$.

$N$-(Ethyloxycarbonyl)-MTA (2d, LH1207): Yield, $62 \mathrm{mg}$ (57\%) obtained from $150 \mathrm{mg}(0.29 \mathrm{mmol})$ of 3d. ${ }^{1} \mathrm{H}$

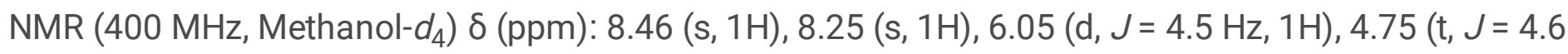
$\mathrm{Hz}, 1 \mathrm{H}), 4.41-4.37(\mathrm{~m}, 1 \mathrm{H}), 4.30(\mathrm{q}, J=4.9 \mathrm{~Hz}, 1 \mathrm{H}), 4.18(\mathrm{q}, J=7.0 \mathrm{~Hz}, 2 \mathrm{H}), 2.86(\mathrm{dd}, J=13.8,5.3 \mathrm{~Hz}$, 1H), $2.78(\mathrm{dd}, J=14.1,5.3 \mathrm{~Hz}, 1 \mathrm{H}), 2.05(\mathrm{~s}, 3 \mathrm{H}), 1.23(\mathrm{t}, J=7.1 \mathrm{~Hz}, 3 \mathrm{H}),{ }^{13} \mathrm{C}$ NMR $\left(100 \mathrm{MHz}\right.$, Methanol- $\left.d_{4}\right)$ $\delta$ (ppm): 152.2, 151.7, 151.1, 149.7, 142.6, 122.3, 88.9, 84.1, 73.3, 72.6, 61.5, 36.0, 15.0, 13.2; HRMS (ESI ${ }^{+}$) $\mathrm{m} / \mathrm{z}$ calcd for $\mathrm{C}_{14} \mathrm{H}_{20} \mathrm{~N}_{5} \mathrm{O}_{5} \mathrm{~S}[\mathrm{M}+\mathrm{H}]^{+}: 370.1180$. Found: 370.1193 .

N-(Propoxycarbonyl)-2',3'-bis(propylcarbonate)-MTA (3e): Yield, $175 \mathrm{mg}$ (46\%) obtained from $200 \mathrm{mg}$ (0.67 mmol) of MTA. ${ }^{1} \mathrm{H}$ NMR (400 MHz, Methanol- $\left.d_{4}\right) \delta(\mathrm{ppm}): 9.16(\mathrm{~s}, 1 \mathrm{H}), 8.77(\mathrm{~s}, 1 \mathrm{H}), 8.20(\mathrm{~s}, 1 \mathrm{H})$, $6.21(\mathrm{~d}, J=5.3 \mathrm{~Hz}, 1 \mathrm{H}), 6.05(\mathrm{t}, J=5.4 \mathrm{~Hz}, 1 \mathrm{H}), 5.59(\mathrm{t}, J=5.0 \mathrm{~Hz}, 1 \mathrm{H}), 4.49(\mathrm{q}, J=5.3 \mathrm{~Hz}, 1 \mathrm{H}), 4.23(\mathrm{t}, J=$ $6.7 \mathrm{~Hz}, 2 \mathrm{H}), 4.17-4.01(\mathrm{~m}, 4 \mathrm{H}), 3.02(\mathrm{dd}, J=14.3,5.3 \mathrm{~Hz}, 1 \mathrm{H}), 2.94(\mathrm{dd}, J=14.3,6.1 \mathrm{~Hz}, 1 \mathrm{H}), 2.12(\mathrm{~s}, 3 \mathrm{H})$, $1.76-1.62(\mathrm{~m}, 6 \mathrm{H}), 0.99-0.88(\mathrm{~m}, 9 \mathrm{H}) ;{ }^{13} \mathrm{C} \mathrm{NMR}\left(100 \mathrm{MHz}\right.$, Methanol- $\left.d_{4}\right) \delta(\mathrm{ppm}): 152.2,151.7,151.1$, $149.6,142.6,122.3,88.9,84.1,73.3,72.6,61.5,36.0,15.0,13.2 ;$ LCMS $\left._{(E S I}\right)$ : $556.6[\mathrm{M}+\mathrm{H}]^{+}$.

$\mathrm{N}$-(Propoxycarbonyl)-MTA (2e, LH1208): Yield, $45 \mathrm{mg}(66 \%)$ obtained from $100 \mathrm{mg}(0.18 \mathrm{mmol})$ of $3 \mathrm{e} .{ }^{1} \mathrm{H}$ NMR (400 MHz, Chloroform- $d$ ) $\delta(\mathrm{ppm}): 8.55(\mathrm{~s}, 1 \mathrm{H}), 8.14(\mathrm{~s}, 1 \mathrm{H}), 5.96(\mathrm{~d}, J=5.3 \mathrm{~Hz}, 1 \mathrm{H}), 4.69$ (t, J = 5.3 $\mathrm{Hz}, 1 \mathrm{H}), 4.42-4.39(\mathrm{~m}, 3 \mathrm{H}), 4.37-4.35(\mathrm{~m}, 1 \mathrm{H}), 4.16(\mathrm{t}, J=6.7 \mathrm{~Hz}, 2 \mathrm{H}), 2.83(\mathrm{dd}, J=12.9,5.0 \mathrm{~Hz}, 1 \mathrm{H})$, $2.78(\mathrm{dd}, J=13.0,4.6 \mathrm{~Hz}, 1 \mathrm{H}), 2.10(\mathrm{~s}, 3 \mathrm{H}), 1.67(\mathrm{dq}, J=14.2,7.2 \mathrm{~Hz}, 2 \mathrm{H}), 0.92(\mathrm{t}, J=7.4 \mathrm{~Hz}, 3 \mathrm{H}) ;{ }^{13} \mathrm{C} \mathrm{NMR}$ (100 MHz, Chloroform- $d$ ) $\delta$ (ppm): 152.4, 151.4, 150.6, 149.4, 141.6, 121.9, 89.5, 84.3, 74.7, 73.2, 67.9, 36.8, 22.1, 16.7, 10.3; HRMS $\left(\mathrm{ESI}^{+}\right) \mathrm{m} / \mathrm{z}$ calcd for $\mathrm{C}_{15} \mathrm{H}_{22} \mathrm{~N}_{5} \mathrm{O}_{5} \mathrm{~S}[\mathrm{M}+\mathrm{H}]^{+}: 384.1336$, found: 384.1350 .

$N$-(2-Methoxyethyoxy)carbonyl-2',3'-bis(2-methoxyethylcarbonate)-MTA (3f): Yield, $200 \mathrm{mg}$ (56\%) obtained from $200 \mathrm{mg}(0.67 \mathrm{mmol})$ of MTA. ${ }^{1} \mathrm{H}$ NMR $(400 \mathrm{MHz}$, Chloroform- $d) \delta(\mathrm{ppm}): 8.79(\mathrm{~s}, 1 \mathrm{H}), 8.69$ 
$(\mathrm{s}, 1 \mathrm{H}), 8.09(\mathrm{~s}, 1 \mathrm{H}), 6.09(\mathrm{~d}, J=5.3 \mathrm{~Hz}, 1 \mathrm{H}), 6.00(\mathrm{t}, J=5.3 \mathrm{~Hz}, 1 \mathrm{H}), 5.53(\mathrm{t}, J=5.0 \mathrm{~Hz}, 1 \mathrm{H}), 4.39(\mathrm{q}, J=5.5$ $\mathrm{Hz}, 2 \mathrm{H}), 4.24(\mathrm{q}, J=4.5,4.0 \mathrm{~Hz}, 3 \mathrm{H}), 4.16(\mathrm{t}, J=4.6 \mathrm{~Hz}, 2 \mathrm{H}), 3.63-3.57(\mathrm{~m}, 2 \mathrm{H}), 3.55(\mathrm{dd}, J=6.8,2.5 \mathrm{~Hz}$, 2H), $3.50-3.45(\mathrm{~m}, 2 \mathrm{H}), 3.31(\mathrm{~d}, J=6.9 \mathrm{~Hz}, 6 \mathrm{H}), 3.25(\mathrm{~s}, 3 \mathrm{H}), 2.93(\mathrm{dd}, J=14.3,5.4 \mathrm{~Hz}, 1 \mathrm{H}), 2.85$ (dd, J = 14.3, 6.2 Hz, 1H), 2.03 (s, 3H); ${ }^{13} \mathrm{C}$ NMR (100 MHz, Chloroform-d) $\delta(p p m): ~ 154.1,153.8,153.1,151.0$, $150.9,149.7,142.2,122.8,86.5,81.8,75.5,75.5,70.4,70.0,69.8,67.9,67.8,64.9,59.0,58.9,36.1$, 16.7; LCMS $\left(\mathrm{ESI}^{+}\right): 604.1[\mathrm{M}+\mathrm{H}]^{+}$.

N-(2-Methoxyethoxycarbonyl)-MTA (2f, LH1209): Yield, 70 mg (62\%) obtained from $170 \mathrm{mg}(0.28 \mathrm{mmol})$ of 3f. ${ }^{1} \mathrm{H}$ NMR (400 MHz, Chloroform-d) $\delta(\mathrm{ppm}): 8.52(\mathrm{~s}, 1 \mathrm{H}), 8.12(\mathrm{~s}, 1 \mathrm{H}), 5.94(\mathrm{~d}, \mathrm{~J}=5.0 \mathrm{~Hz}, 1 \mathrm{H}), 4.69(\mathrm{t}$, $J=5.2 \mathrm{~Hz}, 1 \mathrm{H}), 4.38-4.34(\mathrm{~m}, 1 \mathrm{H}), 4.33-4.30(\mathrm{~m}, 3 \mathrm{H}), 3.58(\mathrm{dd}, J=8.8,4.2 \mathrm{~Hz}, 4 \mathrm{H}), 3.31(\mathrm{~s}, 3 \mathrm{H}), 2.77$ (dq, $J=13.9,6.8,5.6 \mathrm{~Hz}, 2 \mathrm{H}), 2.06$ (s, 3H); ${ }^{13} \mathrm{C}$ NMR (100 MHz, Chloroform-d) $\delta$ (ppm):152.4, 150.9, 150.7,

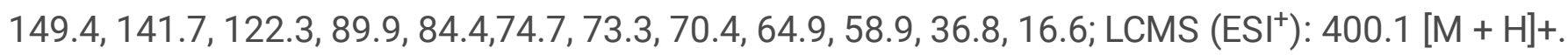

\section{Stability Assay of MTA prodrugs in Phosphate Buffer}

The stock solution of the prodrug (10 mM in DMSO) was diluted 10-fold to give a $1 \mathrm{mM}$ working solution in $10 \%$ DMSO. $100 \mu \mathrm{L}$ of the $1 \mathrm{mM}$ solution was added to $875 \mu \mathrm{L}$ of $50 \mathrm{mM}$ phosphate buffer containing $1 \mathrm{mM}$ EDTA, $\mathrm{pH} 7.4$ and incubated at $37^{\circ} \mathrm{C}$ for 5 minutes in an Eppendorf vial. The vial was then centrifuged for 5 minutes and the supernatant was split into 3 fractions of $292.5 \mu \mathrm{L}$, one for stability in phosphate buffer, one for activation in human liver microsomes and one for activation in mouse liver microsomes.

The fraction for analyzing stability was mixed with $7.5 \mu \mathrm{L}$ of deionized water and incubated at $37^{\circ} \mathrm{C}$ for 3 days. Time points were collected after every $0 \mathrm{~min}, 3 \mathrm{~min}, 5 \mathrm{~min}, 10 \mathrm{~min}, 15 \mathrm{~min}, 30 \mathrm{~min}, 45 \mathrm{~min}, 1 \mathrm{~h}, 3 \mathrm{~h}$, 5 h, 8 h, 10 h, 12 h, 24 h, 48 h, 72 h. At each time point, $20 \mu \mathrm{L}$ aliquots were taken and diluted with $40 \mu \mathrm{L}$ cold acetonitrile and centrifuged for 5 mins. $40 \mu \mathrm{L}$ of the supernatant were taken and diluted with $40 \mu \mathrm{L}$ deionized water and frozen before injecting on the HPLC. The samples were analyzed using a Perkin Elmer Series 2000 HPLC with a Phenomenex Kinetix C18 (50 x $4.6 \mathrm{~mm})$ column and a gradient of 10 $90 \%$ acetonitrile containing $0.1 \%$ TFA in $10 \mathrm{~min}$ at a flowrate of $0.6 \mathrm{~mL} / \mathrm{min}$ was used with detection wavelength of 220 and $254 \mathrm{~nm}$.

\section{In vitro Activation Assay in Human and Mouse Liver Microsomes [18]}

The mouse and human liver microsomes were obtained from Xeno Tech with a protein concentration of $20 \mathrm{mg} / \mathrm{ml}$ and stored in Eppendorf vials at $-70{ }^{\circ} \mathrm{C}$. Stock solution of $1 \mathrm{mM}$ compound in $1 \mathrm{~mL}$ DMSO was diluted 10 -fold to give a $1 \mathrm{mM}$ solution containing $10 \%$ DMSO. $100 \mu \mathrm{L}$ of the $1 \mathrm{mM}$ solution was added to $875 \mu \mathrm{L}$ of $50 \mathrm{mM}$ phosphate buffer containing $1 \mathrm{mM}$ EDTA, pH 7.4 and incubated at $37^{\circ} \mathrm{C}$ for 5 minutes in an Eppendorf vial. The vial was then centrifuged for 5 minutes and the supernatant was split into 3 fractions of $292.5 \mu \mathrm{l}$, one for stability in phosphate buffer, one for activation in human liver microsomes and one for activation in mouse liver microsomes. The fraction for analyzing activation was mixed with $7.5 \mu \mathrm{L}$ of either the human or mouse liver microsomes and incubated at $37^{\circ} \mathrm{C}$ for 2 days. 
Time points were collected after every $0 \mathrm{~min}, 3 \mathrm{~min}, 5 \mathrm{~min}, 10 \mathrm{~min}, 15 \mathrm{~min}, 30 \mathrm{~min}, 45 \mathrm{~min}, 1 \mathrm{~h}, 3 \mathrm{~h}, 5 \mathrm{~h}, 8$ h, 10 h, 12 h, 24 h, and 48 h. At each time point, $20 \mu \mathrm{L}$ aliquots were taken and quenched with $40 \mu \mathrm{L}$ cold acetonitrile and stored on ice for 30 mins before centrifugation for 5 mins. $40 \mu \mathrm{L}$ of the supernatant were taken and diluted with $40 \mu \mathrm{L}$ deionized water and frozen before injecting on the HPLC. The samples were analyzed using a Perkin Elmer Series 2000 HPLC with a Phenomenex Kinetix C18 (50 x 4.6 mm) column and a gradient of $10-90 \%$ acetonitrile containing $0.1 \%$ TFA in $10 \mathrm{~min}$ at a flowrate of $0.6 \mathrm{~mL} / \mathrm{min}$ was used with a detection wavelength of 220 and $254 \mathrm{~nm}$. The half-life was calculated from the first order rate equation of the conversion of prodrug to MTA.

\section{Oxidative Activation Assay in presence of NADPH}

Stock solution (10 mM in DMSO) was diluted 10-fold to give a solution of $1 \mathrm{mM}$ in 10\% DMSO. $100 \mu \mathrm{L}$ of the $1 \mathrm{mM}$ solution was added to $875 \mu \mathrm{L}$ of $50 \mathrm{mM}$ phosphate buffer containing $1 \mathrm{mM}$ EDTA, pH 7.4 and incubated at $37^{\circ} \mathrm{C}$ for 5 minutes in an Eppendorf vial. The vial was then centrifuged for 5 minutes and the supernatant was split into 2 fractions, each of $292.5 \mu \mathrm{L}$, one for oxidative activation in mouse liver microsomes and one for human liver microsomes. Each of the fractions was mixed with $20 \mu \mathrm{L}$ of NADPH and either $20 \mu \mathrm{L}$ of mouse liver microsomes or human liver microsomes and incubated at $37^{\circ} \mathrm{C}$ for $4 \mathrm{~h}$. At the end of incubation, $20 \mu \mathrm{L}$ aliquots were taken and quenched with $40 \mu \mathrm{L}$ cold acetonitrile and stored on ice for 30 mins before centrifugation for 5 mins. $40 \mu \mathrm{L}$ of the supernatant were taken and diluted with $40 \mu \mathrm{L}$ deionized water and frozen prior to HPLC analysis. The samples were analyzed using a Perkin Elmer Series 2000 HPLC with a Phenomenex Kinetix C18 (50 x 4.6 mm) column and a gradient of 10 $90 \%$ acetonitrile containing $0.1 \%$ TFA in $10 \mathrm{~min}$ at a flowrate of $0.6 \mathrm{~mL} / \mathrm{min}$ was used with a detection wavelength of 220 and $254 \mathrm{~nm}$. The half-life was calculated from the first order rate equation of the conversion of prodrug to MTA.

\section{Abbreviations Used}

AMP, adenosine monophosphate; APRT, adenine phosphoribosyltransferase; BNPP, bis(4-nitrophenyl)phosphate; 5-FU, 5-fluorouracil; HLM, human liver microsome; HPLC, high performance liquid chromatography; $I C_{50}$, concentration required to produce $50 \%$ inhibition; LC-MS, liquid chromatographymass spectrometry; MLM, mouse liver microsome; MTA, 5'-methylthioadenosine; MTAP, MTA phosphorylase; NADPH, nicotinamide adenine dinucleotide phosphate; PRPP, phosphoribosyl-1pyrophosphate; 6-TG, 6-thioguanine; TLC, thin layer chromatography.

\section{Declarations}

\section{AUTHOR INFORMATION}

\section{Corresponding Author}

*E-main: LongHu@pharmacy.rutgers.edu 
ORCID

Longqin Hu: 0000-0002-1799-5652

Declaration of Conflicting Interests

The authors declare no competing interests.

\section{References}

1. Casero RA, Jr., Marton LJ. Targeting polyamine metabolism and function in cancer and other hyperproliferative diseases. Nat Rev Drug Discov. 2007;6(5):373-90. doi:10.1038/nrd2243.

2. Avila MaA, García-Trevijano ER, Lu SC, Corrales FJ, Mato JM. Methylthioadenosine. Int J Biochem Cell Biol. 2004;36(11):2125-30. doi:https://doi.org/10.1016/j.biocel.2003.11.016.

3. Lubin M, Lubin A. Selective Killing of Tumors Deficient in Methylthioadenosine Phosphorylase: A Novel Strategy. PLoS ONE. 2009;4(5):e5735. doi:10.1371/journal.pone.0005735.

4. Tang B, Testa JR, Kruger WD. Increasing the therapeutic index of 5-fluorouracil and 6-thioguanine by targeting loss of MTAP in tumor cells. Cancer Biol Ther. 2012;13(11):1082-90. doi:10.4161/cbt.21115.

5. Bertino JR, Waud WR, Parker WB, Lubin M. Targeting tumors that lack methylthioadenosine phosphorylase (MTAP) activity: Current strategies. Cancer Biol Ther. 2011;11(7):627-32. doi:10.4161/cbt.11.7.14948.

6. Kung P-P, Zehnder LR, Meng JJ, Kupchinsky SW, Skalitzky DJ, Johnson MC et al. Design, synthesis, and biological evaluation of novel human 5'-deoxy-5'-methylthioadenosine phosphorylase (MTAP) substrates. Bioorg Med Chem Lett. 2005;15(11):2829-33.

doi:http://dx.doi.org/10.1016/j.bmcl.2005.03.107.

7. Bloom LA, Boritzki TJ, Ogden R, Skalitzky D, Kung P-P, Zehnder L et al., inventors; Agouron Pharmaceuticals, Inc., assignee. Combination therapies for treating methylthioadenosine phosphorylase deficient cells patent US20040043959A1. 2004.

8. Lubin A, Lubin M, inventors; Therapy of tumors and infectious agents deficient in methylthioadenosine phosphorylase patent US 8796241 B2. 2014.

9. Munshi PN, Lubin M, Bertino JR. 6-Thioguanine: A Drug With Unrealized Potential for Cancer Therapy. Oncologist. 2014;19(7):760-5. doi:10.1634/theoncologist.2014-0178.

10. Simile MM, Banni S, Angioni E, Carta G, De Miglio MR, Muroni MR et al. 5\&\#x2032;-

Methylthioadenosine administration prevents lipid peroxidation and fibrogenesis induced in rat liver by carbon-tetrachloride intoxication. Journal of Hepatology. 2001;34(3):386-94. doi:10.1016/S01688278(00)00078-7.

11. Wolford RW, Riscoe MK, Johnson L, Ferro AJ, Fitchen JH. Effect of 5'-methylthioadenosine (a naturally occurring nucleoside) on murine hematopoiesis. Exp Hematol. 1984;12(11):867-71. 
12. Moratti EM, inventor Bioresearch S.P.A., assignee. Pharmaceutical compositions containing 5'-deoxy5 '-methylthioadenosine s-adenosylmethionine and their salts for reducing seborrhea patent US5753213A. 1998.

13. Stramentinoli G, Gennari F, inventors; Bioresearch S.r.l., assignee. Adenosine derivatives of antiinflammatory and analgesic activity, and therapeutic compositions which contain them as their active principle patent US4454122A. 1984.

14. Bloom LA, Boritzki TJ, Kung P-P, Ogden RC, Skalitzky DJ, Zehnder LR et al., inventors; Pfizer Inc., assignee. Combination therapies for treating methylthioadenosine phosphorylase deficient cells patent WO 2003074083 A1. 2003.

15. Hu L. Prodrug Approaches to Drug Delivery. Drug Delivery. 2016. p. 227-71.

16. Yang Y, Aloysius H, Inoyama D, Chen Y, Hu L. Enzyme-mediated hydrolytic activation of prodrugs. Acta Pharm Sin B. 2011;1(3):143-59. doi:https://doi.org/10.1016/j.apsb.2011.08.001.

17. Desmoulin F, Gilard V, Malet-Martino M, Martino R. Metabolism of capecitabine, an oral fluorouracil prodrug: (19)F NMR studies in animal models and human urine. Drug Metab Dispos. 2002;30(11):1221-9. doi:10.1124/dmd.30.11.1221.

18. Shindoh H, Nakano K, Yoshida T, Ishigai M. Comparison of in vitro metabolic conversion of capecitabine to 5-FU in rats, mice, monkeys and humans - toxicological implications. J Toxicol Sci. 2011;36(4):411-22. doi:10.2131/jts.36.411.

\section{Tables}

Table 2. Stability and activation of $N$-(alkyloxy)carbonyl-MTA prodrugs 2 a-f<smiles>[R]OC(=O)Nc1ncnc2c1ncn2[C@@H]1O[C@H]2[C@@H](O)[C@H](CSC)[C@H]1O[C@H]2O</smiles> 


\begin{tabular}{|c|c|c|c|c|}
\hline \multirow[t]{2}{*}{ Compound ID } & \multirow[t]{2}{*}{$\mathbf{R}$} & \multicolumn{3}{|l|}{$t_{1 / 2}(h)^{a}$} \\
\hline & & Buffer & MLM & HLM \\
\hline 2a (LH1201) & $\mathrm{CH}_{2} \mathrm{CH}_{2} \mathrm{CH}_{2} \mathrm{CH}_{2} \mathrm{CH}_{2} \mathrm{CH}_{3}$ & $\begin{array}{l}>72 \\
(<10 \%)\end{array}$ & 1.2 & $\begin{array}{l}>48 \\
(\sim 44 \%)\end{array}$ \\
\hline 2b (LH1205) & $\mathrm{CH}_{2} \mathrm{CH}_{2} \mathrm{CH}_{2} \mathrm{CH}_{3}$ & $\begin{array}{l}>72 \\
(<10 \%)\end{array}$ & 9.4 & $\begin{array}{l}>48 \\
(\sim 19 \%)\end{array}$ \\
\hline 2c (LH1206) & $\mathrm{CH}\left(\mathrm{CH}_{3}\right)_{2}$ & $\begin{array}{l}>72 \\
(<10 \%)\end{array}$ & $\begin{array}{l}>48 \\
(<10 \%)\end{array}$ & $\begin{array}{l}>48 \\
(<10 \%)\end{array}$ \\
\hline 2d (LH1207) & $\mathrm{CH}_{2} \mathrm{CH}_{3}$ & $\begin{array}{l}>72 \\
(<10 \%)\end{array}$ & $\begin{array}{l}>48 \\
(<10 \%)\end{array}$ & $\begin{array}{l}>48 \\
(<10 \%)\end{array}$ \\
\hline 2e (LH1208) & $\mathrm{CH}_{2} \mathrm{CH}_{2} \mathrm{CH}_{3}$ & $\begin{array}{l}>72 \\
(<10 \%)\end{array}$ & 40 & $\begin{array}{l}>48 \\
(<10 \%)\end{array}$ \\
\hline${ }_{(\mathrm{LH}}{ }^{\mathrm{f}} \mathrm{209)}$ & $\mathrm{CH}_{2} \mathrm{CH}_{2} \mathrm{OCH}_{3}$ & $\begin{array}{l}>72 \\
(<10 \%)\end{array}$ & $\begin{array}{l}>48 \\
(\sim 15 \%)\end{array}$ & $\begin{array}{l}>48 \\
(<10 \%)\end{array}$ \\
\hline
\end{tabular}

aThe half lives of prodrugs were measured using HPLC by following their disappearance at $100 \mathrm{mM}$ and $37^{\circ} \mathrm{C}$ in $50 \mathrm{mM}$ Phosphate buffer containing $1 \mathrm{mM}$ EDTA, $\mathrm{pH}=7.4$, in the absence (buffer) or presence of $0.5 \mathrm{mg} / \mathrm{ml}$ mouse liver microsome (MLM) or human liver microsome (HLM). All compounds were followed for $72 \mathrm{~h}$ in phosphate buffer and $48 \mathrm{~h}$ in the presence of MLM or HLM with \% change indicated in brackets at the end of incubation when $<50 \%$ change were observed.

\section{Figures}

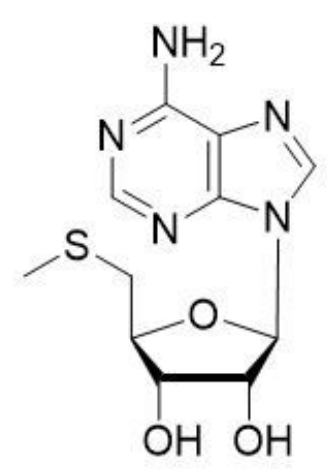

MTA (1)

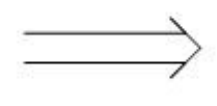

N-(Alkyloxy)carbonyl-MTA (MTA carbamate prodrug, 2a-f) 2a $\mathrm{R}=\mathrm{CH}_{2} \mathrm{CH}_{2} \mathrm{CH}_{2} \mathrm{CH}_{2} \mathrm{CH}_{2} \mathrm{CH}_{3}$

2b $\mathrm{R}=\mathrm{CH}_{2} \mathrm{CH}_{2} \mathrm{CH}_{2} \mathrm{CH}_{3}$

2c $\mathrm{R}=\mathrm{CH}\left(\mathrm{CH}_{3}\right)_{2}$

2d $\mathrm{R}=\mathrm{CH}_{2} \mathrm{CH}_{3}$

2e $\mathrm{R}=\mathrm{CH}_{2} \mathrm{CH}_{2} \mathrm{CH}_{3}$

2f $\mathrm{R}=\mathrm{CH}_{2} \mathrm{CH}_{2} \mathrm{OCH}_{3}$

Figure 1 
Carbamate prodrugs designed to release MTA
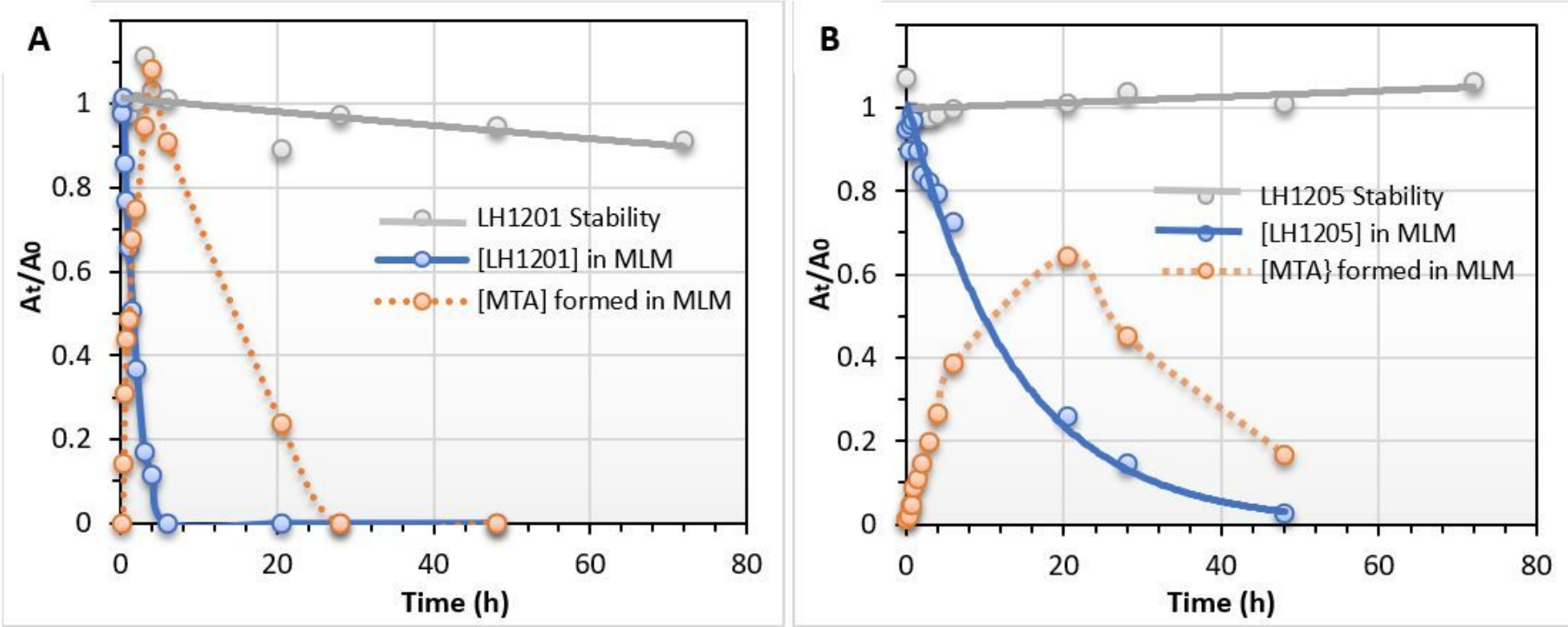

\section{Figure 2}

Stability and activation of N-(alkyloxy)carbonyl-MTA prodrugs 2a (LH1201, A) and 2b (LH1205, B). The relative peak area of each compound was measured using HPLC of aliquots taken at varying time intervals from incubations of each prodrug at $37^{\circ} \mathrm{C}$ in $50 \mathrm{mM}$ Phosphate buffer containing $1 \mathrm{mM}$ EDTA, $\mathrm{pH}=7.4$, in the absence (buffer) or presence of $0.5 \mathrm{mg} / \mathrm{ml}$ mouse liver microsome (MLM). 


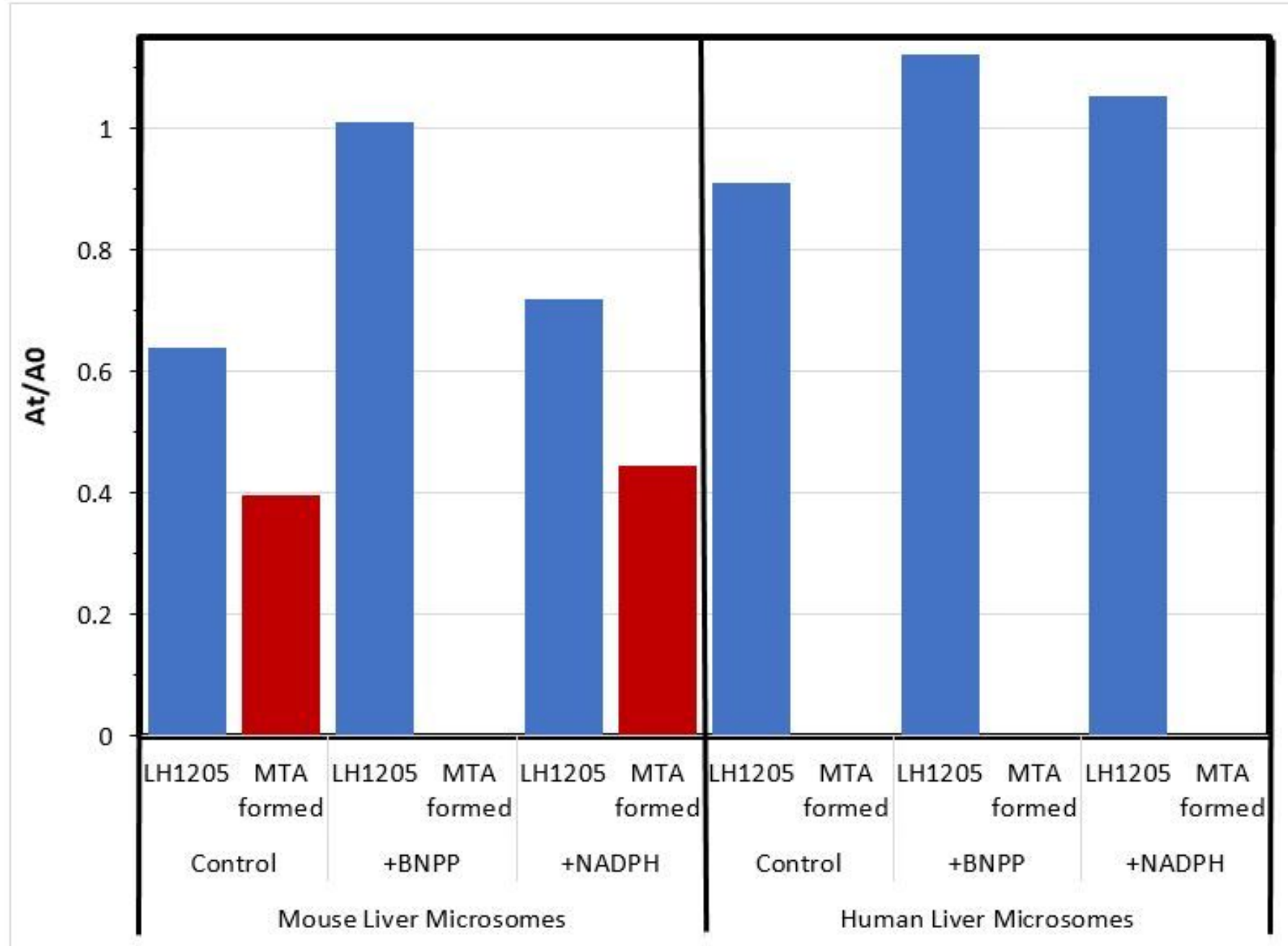

Figure 3

Activation of N-(alkyloxy)carbonyl-MTA prodrug $2 \mathrm{~b}$ after 4-h incubation with mouse or human liver microsomes in the presence of $1 \mathrm{mM}$ BNPP or NADPH. The relative peak area of each compound was measured using HPLC of aliquots taken $4 \mathrm{~h}$ from incubations at $37^{\circ} \mathrm{C}$ in the presence of $0.5 \mathrm{mg} / \mathrm{ml}$ mouse or human liver microsomes +1 mM BNPP or NADPH.

\section{Supplementary Files}

This is a list of supplementary files associated with this preprint. Click to download.

- GA.jpg 